\title{
OPTIMAL WEIGHTED ESTIMATES FOR THE CAUCHY-RIEMANN EQUATION ON ANALYTIC POLYHEDRA
}

\author{
Hong Rae Cho and Jinkee Lee
}

Andersson obtained weighted $L^{p}$ estimates for $\bar{\partial}$ on analytic polyhedra. We provide an example to show why they cannot be improved.

\section{INTRODUCTION}

A bounded domain $\Omega \subset \mathbb{C}^{n}$ is an analytic polyhedron with defining functions $\phi_{j}$ if

$$
\Omega=\left\{z \in \mathbb{C}^{n} ;\left|\phi_{j}(z)\right|<1, \quad j=1, \ldots, N\right\},
$$

where the defining functions $\phi_{j}$ are holomorphic in some neighbourhood of $\bar{\Omega}$. For a multi-index $I \subset\{1, \ldots, N\}$ we let $\sigma_{I}=\left\{z \in \bar{\Omega} ;\left|\phi_{j}(z)\right|=1, j \in I\right\}$.

For $\alpha=\left(\alpha_{1}, \ldots, \alpha_{N}\right)$ let $L_{\alpha}^{p}(\Omega)$ denote the $L^{p}$-space with respect to the weight

$$
d V_{\alpha}=\prod_{j=1}^{N}\left(1-\left|\phi_{j}(z)\right|^{2}\right)^{\alpha_{j}} d V
$$

In [2], Andersson obtained the following $L_{\alpha}^{p}$-estimates for $\bar{\partial}$ on analytic polyhedra. For the estimates he used a generalisation of the Henkin-Ramirez formulas which permits weight factors (see [4]).

THEOREM . Let $\Omega \Subset \mathbb{C}^{n}$ be an analytic polyhedron and let $f$ be a $\bar{\partial}$-closed $(s, q)$ form in $L_{\alpha}^{p}(\Omega), \alpha=\left(\alpha_{1}, \ldots, \alpha_{N}\right), \alpha_{j} \geq 0,1 \leq p<\infty$. If $\wedge_{j \in I} \partial \phi_{j} \neq 0$ on $\sigma_{l}$ for all $|I|<n-q$, then there is a solution $u$ to $\bar{\partial} u=f$ in $L_{\alpha}^{p}(\Omega)$.

The main result of the paper is the sharpness of the $L_{\alpha}^{p}$-estimates for $\bar{\partial}$ in Andersson's theorem. More precisely, we provide a closed $(0,1)$-form $f$ on a polydisc $\Delta^{2}$ in $\mathbb{C}^{2}$ such that if $f \in L_{\alpha}^{p}\left(\Delta^{2}\right)(2 \leq p<\infty, \alpha \geq 0)$ and $v \in L_{\beta}^{r}\left(\Delta^{2}\right)$ is a solution to $\bar{\partial} v=f$, then $\alpha \leq \beta$ and $r \leq p$.

Received 28th October, 1998

The first author was partly supported by the Andong National University and the second author was partly supported by KOSEF postdoctoral fellowship 1998. The second author who is a KOSEF postdoctoral fellow at Andong National University would like to thank Professor E.G. Kwon for his helpful suggestions and fruitful discussions.

Copyright Clearance Centre, Inc. Serial-fee code: 0004-9729/99 \$A2.00+0.00. 


\section{WEIGHTED BERgMAN PROJECTIONS ON THE UNIT POLYDISC}

In this section, we restrict ourselves to the unit polydisc $\Delta^{n}$ in $\mathbb{C}^{n}$.

Let $A_{\alpha}^{p}\left(\Delta^{n}\right)$ denote the weighted Bergman space of holomorphic functions $f$ in $L_{\alpha}^{p}\left(\Delta^{n}\right)$. Then $A_{\alpha}^{p}\left(\Delta^{n}\right)$ is a closed subspace of $L_{\alpha}^{p}\left(\Delta^{n}\right) . L_{\alpha}^{2}\left(\Delta^{n}\right)$ is a Hilbert space with the inner product $(\cdot, \cdot)_{\alpha}$ defined by

$$
(f, g)_{\alpha}=\int_{\Delta^{n}} f(\zeta) \overline{g(\zeta)} \prod_{j=1}^{n}\left(1-\left|\zeta_{j}\right|^{2}\right)^{\alpha_{j}} d V(\zeta)
$$

Since $A_{\alpha}^{2}\left(\Delta^{n}\right)$ is a closed subspace of $L_{\alpha}^{2}\left(\Delta^{n}\right)$, there is an orthogonal projection $P_{\alpha}$ : $L_{\alpha}^{2}\left(\Delta^{n}\right) \rightarrow A_{\alpha}^{2}\left(\Delta^{n}\right)$. It can be shown that

$$
P_{\alpha} f(z)=C_{\alpha} \int_{\Delta^{n}} f(\zeta) \prod_{j=1}^{n} \frac{\left(1-\left|\zeta_{j}\right|^{2}\right)^{\alpha_{j}}}{\left(1-\overline{\zeta_{j}} z_{j}\right)^{\alpha_{j}+2}} d V(\zeta), \quad z \in \Delta^{n}
$$

where $C_{\alpha}=\prod_{j=1}^{n}\left(\alpha_{j}+1\right)[1,2,3,6]$. We call the projection $P_{\alpha}$ by the weighted Bergman projection.

Using [5, Proposition 1.4.10] repeatly, we can obtain the following result.

LEMMA 2.1. For $\alpha \geq 0$, let

$$
K_{\alpha}(\zeta, z)=C_{\alpha} \prod_{j=1}^{n} \frac{\left(1-\left|\zeta_{j}\right|^{2}\right)^{\alpha_{j}}}{\left(1-\overline{\zeta_{j}} z_{j}\right)^{\alpha_{j}+2}}
$$

and

$$
h(z)=\prod_{j=1}^{n}\left(1-\left|z_{j}\right|^{2}\right)^{-c_{j}} .
$$

(i) If $0<c_{j}<1+\alpha_{j}, j=1, \ldots, n$, then

$$
\int_{\zeta \in \Delta^{n}}\left|K_{\alpha}(\zeta, z)\right| h(\zeta) d V(\zeta) \lesssim h(z), \quad z \in \Delta^{n}
$$

(ii) If $0<c_{j}+\alpha_{j}<1+\alpha_{j}, j=1, \ldots, n$, then

$$
\int_{z \in \Delta^{n}}\left|K_{\alpha}(\zeta, z)\right| h(z) d V(z) \lesssim h(\zeta), \quad \zeta \in \Delta^{n}
$$


By the mean value property, it follows that

$$
\int_{0}^{2 \pi} \rho e^{i \theta} h\left(\zeta_{1}, \rho e^{i \theta}\right) d \theta=2 \pi \cdot 0 \cdot h\left(\zeta_{1}, 0\right)=0
$$

Thus we have $(h, u)_{\beta}=0$, that is, $u$ is orthogonal to all $L_{\beta}^{2}$ holomorphic functions on $\Delta^{2}$. If $v \in L_{\beta}^{p}\left(\Delta^{2}\right)$ is a solution to $\bar{\partial} v=f$ in $\Delta^{2}$, then by Theorem 2.2, $u=v-P_{\beta}(v)$ would be in $L_{\beta}^{p}\left(\Delta^{2}\right)$. This is impossible. Hence there is no solution $v$ in $L_{\beta}^{p}\left(\Delta^{2}\right)$ to the equation $\bar{\partial} v=f$ for $\beta<\alpha$.

If we take $d=\left(2+\alpha_{1}\right) / r$, then it also follows by a similar process to the above that every solution $v$ to $\bar{\partial} v=f$ is not in $L_{\alpha}^{r}\left(\Delta^{2}\right)$ for $p<r$.

\section{REFERENCES}

[1] K. Adachi, M. Andersson and H.R. Cho, ' $L^{p}$ and $H^{p}$ extensions of holomorphic functions from subvarieties of analytic polyhedra', Pacific J. Math. (to appear).

[2] M. Andersson, ' $L_{p}$ estimates for the $\bar{\partial}$-equation in analytic polyhedra in Stein manifolds', in Several complex variables (Stockholm, 1987/1988), Math. Notes 38 (Princeton Univ. Press, Princeton, NJ, 1993), pp. 34-47.

[3] B. Berndtsson, 'Weighted integral formulas', in Several complex variables (Stockholm, 1987/1988), Math. Notes 38 (Princeton Univ. Press, Princeton, NJ, 1993), pp. 160-187.

[4] B. Berndtsson and M. Andersson, 'Henkin-Ramirez formulas with weight factors', Ann. Inst. Fourier 32 (1982), 91-110.

[5] W. Rudin, Function theory in the unit ball of $\mathbb{C}^{n}$ (Springer-Verlag, Berlin, Heidelberg, New York, 1980).

[6] K. Zhu, 'Weighted Bergman projections on the polydisc', Houston J. Math. 20 (1994), 275-292.

Department of Mathematics Education

Andong National University

Andong 760-749

Korea

e-mail: chohr@andong.ac.kr 